CYBERNETICS AND INFORMATION TECHNOLOGIES • Volume 18, No 5

Special Thematic Issue on Optimal Codes and Related Topics

\title{
New Results on Binary Codes Obtained by Doubling Construction
}

\author{
Alexander A. Davydov ${ }^{1}$, Stefano Marcugini ${ }^{2}$, Fernanda Pambianco ${ }^{2}$ \\ ${ }^{1}$ Kharkevich Institute for Information Transmission Problems, Russian Academy of Sciences, Bol'shoi \\ Karetnyi pereulok 19, Moscow 127051, Russian Federation \\ ${ }^{2}$ Dipartimento di Matematica e Informatica, Università degli Studi di Perugia, Via Vanvitelli 1, Perugia \\ 06123, Italy \\ E-mails:adav@iitp.ru stefano.marcugini@unipg.it fernanda.pambianco@unipg.it
}

\begin{abstract}
Binary codes created by doubling construction, including quasi-perfect ones with distance $d=4$, are investigated. All $\left[17 \cdot 2^{r-6}, 17 \cdot 2^{r-6}-r, 4\right]$ quasi-perfect codes are classified. Weight spectrum of the codes dual to quasi-perfect ones with $d=4$ is obtained. The automorphism group $\mathrm{Aut}(C)$ of codes obtained by doubling construction is studied. A subgroup of $A u t(C)$ is described and it is proved that the subgroup coincides with $A u t(C)$ if the starting matrix of doubling construction has an odd number of columns. (It happens for all quasi-perfect codes with $d=4$ except for Hamming one.) The properness and t-properness for error detection of codes obtained by doubling construction are considered.
\end{abstract}

Keywords: Linear binary codes, doubling construction, quasi-perfect codes, automorphism group of a code, proper and t-proper codes.

\section{Introduction}

Let an $[n, n-r, d]$ code be a linear binary code of length $n$, redundancy $r$, and minimum distance $d$. A code with $d=4$ is quasi-perfect if its covering radius is equal to 2. Addition of any column to a parity check matrix of a quasi-perfect code decreases the code distance. A parity check matrix of a quasi-perfect $[n, n-r, 4]$ code can be treated as a complete $n$-cap in the projective space $\mathrm{PG}(r-1,2)$ of dimension $r-1$. A cap in $\operatorname{PG}(N, 2)$ is a set of points no three of which are collinear. A cap is complete if no point can be added to it.

Observation 1. An arbitrary $[n, n-r, 4]$ code is either a quasi-perfect code or the shortening of some quasi-perfect code with $d=4$ and redundancy $r$.

So, studying quasi-perfect codes is important. The $\left[2^{r-1}, 2^{r-1}-r, 4\right]$ extended Hamming code is deeply investigated. The $\left[5 \cdot 2^{r-4}, 5 \cdot 2^{r-4}-r, 4\right]$ Panchenko code $[1,2,6,7,15]$ draws attention as in it the number of weight 4 codewords is small and, in a number of cases, the smallest possible among all codes with $d=4$. This essentially increases the error detection capability of Panchenko code. Nevertheless, 
Panchenko code is studied insufficiently. The same can be said about other quasiperfect $[n, n-r, 4]$ codes (not about Hamming one).

Observation 2 [6]. All quasi-perfect [ $n, n-r, 4]$ codes of length $n \geq 2^{r-2}+2$ can be described by doubling construction (see Equation (1) below).

So, it is appropriate to study quasi-perfect $[n, n-r, 4]$ codes from the point of view of doubling construction. Such researches were done, for instance, in $[1,2,6,7]$. In this work we continue investigations of codes created by doubling construction, including quasi-perfect ones.

In Section 2, we describe doubling construction and, basing on the results of [6], give a general description of a parity check matrix for a whole class of quasi-perfect binary codes with distance 4 . Also, we classify all quasi-perfect $[17,17-6,4]$ codes and thereby all quasi-perfect $\left[n_{r}, n_{r}-r, 4\right]$ codes with $n_{r}=17 \cdot 2^{r-6}, r \geq 6$.

In Section 3, we prove a general theorem on weight spectrum of the code dual to quasi-perfect one and obtain all these spectra for quasi-perfect $\left[n_{r}, n_{r}-r, 4\right]$ codes with $n_{r}=2^{r-2}+2^{r-2-g}, g=2,3,4, r \geq g+2$.

In Section 4, the Automorphism group $\operatorname{Aut}(C)$ of codes obtained by doubling construction is investigated. We describe a subgroup $G$ of $\operatorname{Aut}(C)$ and prove that if the starting matrix of doubling construction has an odd number of columns then $G=\operatorname{Aut}(C)$. It happens for all quasi-perfect codes with $d=4$ except for Hamming one.

In Section 5, the properness and t-properness for error detection of codes, obtained by doubling construction, is considered. We use the results of this work and papers $[3,8-11]$.

Some results of this work were briefly presented in [5].

\section{Doubling construction and classification of binary quasi-perfect codes with distance 4}

For a code with redundancy $r$ we introduce the following notations: $n_{r}$ is length of the code, $H_{r}$ is its parity check matrix of size $r \times n_{r}$, and $d_{r}$ is code distance.

Definition 1. Doubling construction creates a parity check matrix $H_{r}$ of an $\left[n_{r}, n_{r}-r, d_{r}\right]$ code from a parity check matrix $H_{r-1}$ of an $\left[n_{r-1}, n_{r-1}-(r-1), d_{r-1}\right]$ code as follows:

$$
H_{r}=\left[\begin{array}{c|c}
0 \ldots 0 & 1 \ldots 1 \\
--- & --- \\
H_{r-1} & H_{r-1}
\end{array}\right] .
$$

By (1), $n_{r}=2 n_{r-1}$. Also, if $d_{r-1}=3$ then $d_{r}=3$; if $d_{r-1} \geq 4$ then $d_{r}=4$. Doubling construction is called also Plotkin construction, see [6] and the references therein.

Let us define matrices $M, S$, and $\Omega$ as 


$$
M=\left[\begin{array}{l}
01 \\
11
\end{array}\right], S=\left[\begin{array}{l}
10001 \\
01001 \\
00101 \\
00011
\end{array}\right], \Omega=\left[\begin{array}{ll}
00000 & 1111 \\
10001 & 0000 \\
01001 & 1001 \\
00101 & 0101 \\
00011 & 0011
\end{array}\right] .
$$

The matrix $S$ (respectively $\Omega$ ) can be treated as a parity check matrix of the $\left[2^{2}+1,1,5\right]$ perfect repetition code (resp. $\left[2^{3}+1,4,4\right]$ quasi-perfect code). By $[6$, Lemma 10], there exists only one (up to equivalence) $\left[2^{3}+1,4,4\right]$ quasi-perfect code; moreover, the parity check matrix of this code can be presented in the form $\Omega$.

From the results of the paper [6], we have a general description of a parity check matrix for a whole class of quasi-perfect codes with distance 4 .

Theorem 1 [6]. (i) Let $n_{r} \geq 2^{r-2}+2, r \geq 5$, and let an [ $\left.n_{r}, n_{r}-\mathrm{r}, 4\right]$ code be quasiperfect. Then length $n_{r}$ can take any value from the sequence

$$
\text { (3) } \quad n_{r}=2^{r-2}+2^{r-2-g}=\left(2^{g}+1\right) 2^{r-2-g} \text { for } g=0,2,3,4,5, \ldots, r-3 \text {. }
$$

Moreover, $n_{r}$ may not take any other value that is not listed in (3). Also, for each $g=0,2,3,4,5, \ldots, r-3$, there exists an $\left[n_{r}, n_{r}-r, 4\right]$ quasi-perfect code with $n_{r}=2^{r-2}+2^{r-2-g}$.

(ii) Let $n_{r}=2^{r-2}+2^{r-2-g}=\left(2^{g}+1\right) 2^{r-2-g}, g \in\{0,2,3,4,5, \ldots, r-3\}, r \geq 5$, and let an $\left[n_{r}, n_{r}-r, 4\right]$ code be quasi-perfect. Then a parity check matrix $H_{r}$ of this code can be presented in the form

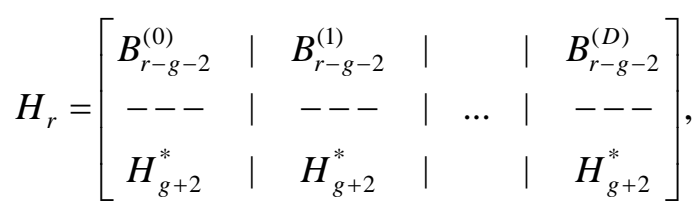

where $D=2^{r-g-2}-1, B_{r-g-2}^{(j)}=\left[b_{r-g-2}^{(j)} \ldots b_{r-g-2}^{(j)}\right]$ is the $(r-g-2) \times\left(2^{g}+1\right)$ matrix of identical columns $b_{r-g-2}^{(j)}$ every of which is the $(r-g-2)$-positional binary representation of the integer $j$ (with the most significant bit at the top position), $H_{0+2}^{*}=M, H_{2+2}^{*}=S, H_{3+2}^{*}=\Omega, H_{g+2}^{*}$ is a parity check matrix of a quasi-perfect $\left[2^{g}+1,2^{g}+1-(g+2), 4\right]$ code if $g \geq 4$.

The $\left[2^{r-1}, 2^{r-1}-r, 4\right]$ code (with starting matrix $M$ ) is the extended Hamming code. The $\left[5 \cdot 2^{r-4}, 5 \cdot 2^{r-4}-r, 4\right]$ code (with starting matrix $S$ ) is the Panchenko code $\Pi_{r}$ proposed in [15], see also $[1,2,6,7]$. The parity check matrix of $\Pi_{r}$ is the matrix $H_{r}$ of (4) with $g=2, D=2^{r-4}-1, H_{g+2}^{*}=S$. We denote by $W_{r}$ the $\left[9 \cdot 2^{r-5}, 9 \cdot 2^{r-5}-r, 4\right]$ code (with starting matrix $\Omega$ ).

By Theorem 1, all quasi-perfect $\left[n_{r}, n_{r}-r\right.$, 4] codes with $g=0,2,3$, and, respectively, $n_{r}=2^{r-1}, n_{r}=5 \cdot 2^{r-4}$, and $n_{r}=9 \cdot 2^{r-4}$, are classified.

Corollary 1. For $g \geq 4$ and $n_{r}=2^{r-2}+2^{r-2-g}$, in order to classify all quasi-perfect $\left[n_{r}, n_{r}-r, 4\right]$ codes, it is sufficient to classify all quasi-perfect $\left[2^{g}+1,2^{g}+1-\right.$ $(\mathrm{g}+2), 4]$ codes. 
In order to classify $\left[2^{4}+1,2^{4}+1-(4+2), 4\right]$ codes, we (similarly to [6, Equation (18)]) introduce a $(g+2) \times\left(2^{g}+1\right)$ matrix

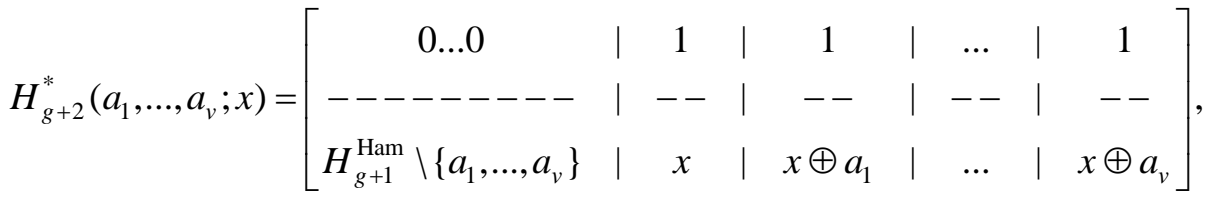

where $a_{i}$ and $x$ are $(g+1)$-positional distinct columns; the entry $H_{g+1}^{\mathrm{Ham}} \backslash\left\{a_{1}, \ldots, a_{v}\right\}$ notes the $(g+1) \times\left(2^{g}-v\right)$ matrix obtained by removing of the columns $a_{1}, \ldots, a_{v}$ from the parity check matrix of the $\left[2^{g}, 2^{g}-(g+1), 4\right]$ extended Hamming code; $\oplus$ means the bit-by-bit sum of binary columns modulo two; $v$ is a parameter.

Conjecture 1 [6, Remark 5].

(i) There exist exactly 5 distinct (up to equivalence) quasi-perfect $\left[2^{4}+1,2^{4}+1-(4+2), 4\right]$ codes.

(ii) Parity check matrices of these codes can be presented in the form

$$
H_{4+2}^{*}\left(a_{1}, a_{2}, \ldots, a_{v} ; x\right) \text { with } v=1,3,4,5,6 \text {, }
$$

where 5-positional columns $a_{1}, a_{2}, \ldots, a_{v}$ are linearly independent for $v \leq 5$, columns $a_{1}, a_{2}, a_{3}, a_{4}, a_{5}$ are linear independent for $v=6$.

Note that the order of columns $a_{1}, a_{2}, \ldots, a_{v}$ does not influence the properties of the matrix $H_{4+2}^{*}\left(a_{1}, a_{2}, \ldots, a_{v} ; x\right)$. Therefore, for $v=6$ any quintuplet of columns from the set $\left\{a_{1}, a_{2}, a_{3}, a_{4}, a_{5}, a_{6}\right\}$ must be linearly independent. It is possible, for instance, if the columns $a_{1}, a_{2}, a_{3}, a_{4}, a_{5}$ are linearly independent and also $a_{6}=a_{1} \oplus a_{2} \oplus a_{3} \oplus a_{4} \oplus a_{5}$.

Conjecture 1(i) is proved in $[4,12]$ by exhaustive computer search.

Proposition $1[4,12]$. There exist exactly 5 distinct (up to equivalence) quasiperfect $[17,11,4]$ codes.

In this work, we prove Conjecture 1(ii) for specified columns $a_{i}$ and $x$. We put

$$
a_{1}=(10000)^{\mathrm{T}}, a_{2}=(10001)^{\mathrm{T}}, a_{3}=(10010)^{\mathrm{T}}, a_{4}=(10100)^{\mathrm{T}},
$$

$$
a_{5}=(11000)^{\mathrm{T}}, x=(11111)^{\mathrm{T}}, a_{6}=a_{1} \oplus a_{2} \oplus a_{3} \oplus a_{4} \oplus a_{5}=(11111)^{\mathrm{T}}, x^{\prime}=(11110)^{\mathrm{T}} \text {. }
$$

Note that, in (6), the columns $a_{1}, a_{2}, a_{3}, a_{4}, a_{5}$ are linearly independent.

Let us define the matrices $\Phi_{1}, \ldots, \Phi_{5}$ as follows:

$$
\begin{gathered}
\Phi_{1}=H_{4+2}^{*}\left(a_{1} ; x\right), \Phi_{2}=H_{4+2}^{*}\left(a_{1}, a_{2}, a_{3} ; x\right), \Phi_{3}=H_{4+2}^{*}\left(a_{1}, a_{2}, a_{3}, a_{4} ; x\right), \\
\Phi_{4}=H_{4+2}^{*}\left(a_{1}, a_{2}, a_{3}, a_{4}, a_{5} ; x\right), \Phi_{5}=H_{4+2}^{*}\left(a_{1}, a_{2}, a_{3}, a_{4}, a_{5}, a_{6} ; x^{\prime}\right),
\end{gathered}
$$

where $a_{i}, x$, and $x^{\prime}$ are taken from (6).

By (5)-(7), we have

$\Phi_{1}=\left[\begin{array}{lll}0000000 & 00000000 & 11 \\ 1111111 & 11111111 & 10 \\ 0000000 & 11111111 & 11 \\ 0001111 & 00001111 & 11 \\ 0110011 & 00110011 & 11 \\ 1010101 & 01010101 & 11\end{array}\right], \quad \Phi_{2}=\left[\begin{array}{lll}00000 & 00000000 & 1111 \\ 11111 & 11111111 & 1000 \\ 00000 & 11111111 & 1111 \\ 01111 & 00001111 & 1111 \\ 10011 & 00110011 & 1110 \\ 10101 & 01010101 & 1101\end{array}\right]$,


(9) $\Phi_{3}=\left[\begin{array}{lll}0000 & 00000000 & 11111 \\ 1111 & 11111111 & 10000 \\ 0000 & 11111111 & 11111 \\ 0111 & 00001111 & 11110 \\ 1011 & 00110011 & 11101 \\ 1101 & 01010101 & 11011\end{array}\right], \Phi_{4}=\left[\begin{array}{lll}0000 & 0000000 & 111111 \\ 1111 & 1111111 & 100000 \\ 0000 & 1111111 & 111110 \\ 0111 & 0001111 & 111101 \\ 1011 & 0110011 & 111011 \\ 1101 & 1010101 & 110111\end{array}\right]$,
$\Phi_{5}=\left[\begin{array}{llll}0000 & 000000 & 1111111 \\ 1111 & 111111 & 1000000 \\ 0000 & 111111 & 1111100 \\ 0111 & 000111 & 1111010 \\ 1011 & 011001 & 1110110 \\ 1101 & 101010 & 0010001\end{array}\right]$.

Proposition 2. All matrices $\Phi_{1}, \ldots, \Phi_{5}$ are non equivalent to each other and every matrix is a parity check matrix of a $[17,11,4]$ quasi-perfect code.

Proof: We checked the assertion by computer.

By Propositions 1 and 2, the following theorem is proved.

Theorem 2. The five codes with the parity check matrices $\Phi_{1}, \ldots, \Phi_{5}$ give the whole list of all distinct, up to equivalence, $\left[2^{4}+1,2^{4}+1-(4+2), 4\right]$ quasi-perfect codes.

Now, by Corollary 1 , we can say that all quasi-perfect $\left[n_{r}, n_{r}-r, 4\right]$ codes with $n_{r}=17 \cdot 2^{r-6}, r \geq 6$, are classified.

\section{Dual weight spectrum of codes obtained by doubling construction}

For a code $C$, let $A_{w}$ (respectively $A_{w}^{\perp}$ ) be the number of codewords of weight $w$ in $C$ (respectively in the dual code $C^{\perp}$ ). Usually, the code is clear by context. To emphasize the code we can write $A_{w}(C)$ or $A_{w}^{\perp}(C)$.

Theorem 3. Let $g \geq 2$ and let $\left\{A_{w}^{\perp}\left(T_{g+2}\right), w=0,1, \ldots, 2^{g}+1\right\}$ be the weight spectrum of the code dual to the starting $\left[2^{g}+1,2^{g}+1-(g+2), d\right]$ code $T_{g+2}$ with the parity check matrix $H_{g+2}^{*}$ of the construction (4). Then the weight spectrum of the code dual to the resultant $\left[\left(2^{g}+1\right) 2^{r-2-g},\left(2^{g}+1\right) 2^{r-2-g}-r, 4\right]$ code $C_{r}$ with the parity check matrix $H_{r}$ of (4) is as follows:

$$
\begin{gathered}
A_{w 2^{r-2-g}}^{\perp}\left(C_{r}\right)=A_{w}^{\perp}\left(T_{g+2}\right), w=0,1, \ldots, 2^{g}+1, \\
A_{\left(2^{g}+1\right) 2^{r-3-g}\left(C_{r}\right)=2^{r}-2^{g+2},}^{\perp} \\
A_{u}^{\perp}\left(C_{r}\right)=0, u \notin\left\{0 \cdot 2^{r-2-g}, 1 \cdot 2^{r-2-g}, \ldots,\left(2^{g}+1\right) 2^{r-2-g}\right\} \cup\left\{\left(2^{g}+1\right) 2^{r-3-g}\right\} .
\end{gathered}
$$

Proof: We consider the matrix $H_{r}$ of (4) as a generator matrix of the dual code. If a codeword of the dual code is created without the inclusion of the top $r-g-2$ rows 
(i.e., without matrices $B_{r-g-2}^{(j)}$, then its weight is equal to the weight of the corresponding word formed from rows of matrix $H_{g+2}^{*}$ multiplied by $D+1=2^{r-g-2}$. This explains the term $A_{w 2^{r-2-g}}^{\perp}\left(C_{r}\right)=A_{w}^{\perp}\left(T_{g+2}\right)$. If at least one of the top $r-g-2$ rows of $H_{r}$ in (4) is used for creating a word of the dual code, then the weight of this word is equal to $\left(2^{g}+1\right) 2^{r-3-g}$. The number of such words is $2^{r}-2^{g+2}$.

Let $V_{r, j}$ be the $\left[17 \cdot 2^{r-6}, 17 \cdot 2^{r-6}-r, 4\right]$ code with the parity check matrix $H_{r}$ of (4) where $g=4, H_{g+2}^{*}=H_{4+2}^{*}=\Phi_{j}, D=2^{r-6}-1, j=1, \ldots, 5$.

Proposition 3. For the $\left[n_{r}, n_{r}-r, 4\right]$ quasi-perfect codes $\Pi_{r}, W_{r}$, and $V_{r, 1}, \ldots$, $V_{r, 5}$, the weight spectrum of the nonzero weights of the dual codes is as follows:

$$
\begin{aligned}
& \Pi_{r}, n_{r}=5 \cdot 2^{r-4}: \mathrm{A}_{2 \cdot 2^{r-4}}^{\perp}=10, A_{5 \cdot 2^{r-5}}^{\perp}=2^{r}-2^{4}, A_{4 \cdot 2^{r-4}}^{\perp}=5, \\
& W_{r}, n_{r}=9 \cdot 2^{r-5}: A_{2 \cdot 2^{r-5}}^{\perp}=1, A_{4 \cdot 2^{r-5}}^{\perp}=21, A_{9 \cdot 2^{r-6}}^{\perp}=2^{r}-2^{5} \text {, }
\end{aligned}
$$

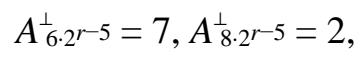

$$
\begin{aligned}
& V_{r, 1}, n_{r}=17 \cdot 2^{r-6}: A_{2 \cdot 2^{r-6}}^{\perp}=1, A_{8 \cdot 2^{r-6}}^{\perp}=45, A_{17 \cdot 2^{r-7}}^{\perp}=2^{r}-2^{6} \text {, }
\end{aligned}
$$

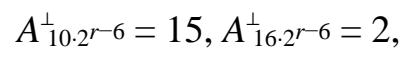

$$
\begin{aligned}
& V_{r, 2}, n_{r}=17 \cdot 2^{r-6}: A_{4 \cdot 2^{r-6}}^{\perp}=1, A_{6 \cdot 2^{r-6}}^{\perp}=3, A_{8 \cdot 2^{r-6}}^{\perp}=42, A_{17 \cdot 2^{r-7}}^{\perp}=2^{r}-2^{6} \text {, }
\end{aligned}
$$

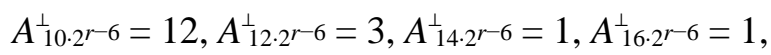

$$
\begin{aligned}
& V_{r, 3}, n_{r}=17 \cdot 2^{r-6}: A_{5 \cdot 2^{r-6}}^{\perp}=2, A_{7 \cdot 2^{r-6}}^{\perp}=8, A_{8 \cdot 2^{r-6}}^{\perp}=30, A_{17 \cdot 2^{r-7}}^{\perp}=2^{r}-2^{6} \text {, } \\
& A_{9 \cdot 2^{r-6}}^{\perp}=12, A_{11 \cdot 2^{r-6}}^{\perp}=8, A_{13 \cdot 2^{r-6}}^{\perp}=2, A_{16 \cdot 2^{r-6}}^{\perp}=1 \text {; } \\
& V_{r, 4}, n_{r}=17 \cdot 2^{r-6}: A_{6 \cdot 2^{r-6}}^{\perp}=6, A_{8 \cdot 2^{r-6}}^{\perp}=40, A_{17 \cdot 2^{r-7}}^{\perp}=2^{r}-2^{6} \text {, } \\
& A_{10 \cdot 2^{r-6}}^{\perp}=10, A_{12 \cdot 2^{r-6}}^{\perp}=6, A_{16 \cdot 2^{r-6}}^{\perp}=1 ; \\
& V_{r, 5}, n_{r}=17 \cdot 2^{r-6}: A_{7 \cdot 2^{r-6}}^{\perp}=16, A_{8 \cdot 2^{r-6}}^{\perp}=30, A_{17 \cdot 2^{r-7}}^{\perp}=2^{r}-2^{6} \text {, } \\
& A_{11 \cdot 2^{r-6}}^{\perp}=16, A_{16 \cdot 2^{r-6}}^{\perp}=1 .
\end{aligned}
$$

Proof: By computer search, we obtained the following dual weight spectra of the nonzero weights of the starting $\left[n_{g+2}, n_{g+2}-(g+2), 4\right]$ quasi-perfect codes with the parity check matrices $S, \Omega, \Phi_{1}, \ldots, \Phi_{5}$ :

$$
\begin{gathered}
S, n_{g+2}=5: A_{2}^{\perp}=10, A_{4}^{\perp}=5 ; \\
\Omega, n_{g+2}=9: A_{2}^{\perp}=1, A_{4}^{\perp}=21, A_{6}^{\perp}=7, A_{8}^{\perp}=2, \\
\Phi_{1}, n_{g+2}=17: A_{2}^{\perp}=1, A_{8}^{\perp}=45, A_{10}^{\perp}=15, A_{16}^{\perp}=2, \\
\Phi_{2}, n_{g+2}=17: A_{4}^{\perp}=1, A_{6}^{\perp}=3, A_{8}^{\perp}=42, A_{10}^{\perp}=12, A_{12}^{\perp}=3, A_{14}^{\perp}=1, A_{16}^{\perp}=1, \\
\Phi_{3}, n_{g+2}=17: A_{5}^{\perp}=2, A_{7}^{\perp}=8, A_{8}^{\perp}=30, A_{9}^{\perp}=12, A_{11}^{\perp}=8, A_{13}^{\perp}=2, A_{16}^{\perp}=1, \\
\Phi_{4}, n_{g+2}=17: A_{6}^{\perp}=6, A_{8}^{\perp}=40, A_{10}^{\perp}=10, A_{12}^{\perp}=6, A_{16}^{\perp}=1, \\
\Phi_{5}, n_{g+2}=17: A_{7}^{\perp}=16, A_{8}^{\perp}=30, A_{11}^{\perp}=16, A_{16}^{\perp}=1 .
\end{gathered}
$$

Now we use Theorem 3. 


\section{The automorphism group of codes created by doubling construction}

In this section we investigate the properties of the automorphism group of the codes obtained applying doubling construction.

Definition 2. The permutations of coordinate places which send a code $C$ into itself form the code automorphism group of $C$, denoted by $\operatorname{Aut}(C)$.

A code and its dual have the same automorphism group.

Theorem 4 [14, Chapter 8, Problem 29]. $\operatorname{Aut}(C)=\operatorname{Aut}\left(C^{\perp}\right)$.

Let $C$ be an $[n, n-r, d]$ code, let $\pi \in \operatorname{Aut}(C)$, and let $g_{1}, \ldots, g_{n-r}$ be the rows of a generator matrix $G$ of the code $C$. Then $\pi\left(g_{1}\right), \ldots, \pi\left(g_{n-r}\right)$ is a basis of $C$ too. Therefore a change of basis matrix belonging to the general linear group $\operatorname{GL}(n-r, 2)$ corresponds to $\pi$.

On the other hand, we can consider the columns $c_{j}$ of $G$ as points of the projective space $\operatorname{PG}(n-r-1,2)$. Let $K \in \mathrm{GL}(n-r, 2)=\operatorname{PGL}(n-r, 2)$ belong to the stabilizer group of the set $\Sigma=\left\{c_{j}\right\}_{j=1, \ldots, n}$, i.e., $K c_{j} \in \Sigma, \forall j \in\{1, \ldots, n\}$. Then $K$ induces a permutation of the coordinate places and therefore preserves the weight of each codeword. Then, by [14, Chapter 8, Problem 33], if no coordinate of $C$ is always zero, $K$ corresponds to a permutation $\pi \in \operatorname{Aut}(C)$.

From the discussion above and Theorem 4, we can represent $\operatorname{Aut}(C)$ as the stabilizer group of the columns of its parity check matrix $H_{r}$ treated as points of $\mathrm{PG}(r-1,2)$. We will denote $\operatorname{Aut}(C)$ also as $\operatorname{Aut}\left(H_{r}\right)$.

Lemma 1. The $r \times 2^{r-s} n_{s}$ matrix $H_{r}$, obtained from a starting $s \times n_{s}$ matrix $H_{s}$ applying doubling construction $r-s$ times, has the form

$$
H_{r}=\left[\begin{array}{c|c|c}
b_{r-s}^{(0)} \ldots b_{r-s}^{(0)} & b_{r-s}^{(1)} \ldots b_{r-s}^{(1)} & b_{r-s}^{\left(2^{\ell}-1\right)} \ldots b_{r-s}^{\left(2^{\ell}-1\right)} \\
----- & ----- & ------ \\
h_{1} \ldots h_{n_{S}} & h_{1} \ldots h_{n_{s}} & h_{1} \ldots h_{n_{s}}
\end{array}\right],
$$

where $\ell=r-s, h_{j}$ is the $j$-th $s$-positional column of $H_{s}$, and $b_{r-s}^{(i)}$ is the $(r-s)$-positional binary representation of the integer $i$.

Proof: By induction on $r-s$.

Now we describe a subgroup of $\operatorname{Aut}(C)$. Let $Z_{\ell, m}$ be the $\ell \times m$ matrix with all entries equal to 0 and let $T_{\ell, m}$ be any $\ell \times m$ binary matrix. We denote by $\Gamma_{r}$ the following set of matrices:

$$
\left.\Gamma_{r}=\left\{\begin{array}{c|c}
K_{r-s} & T_{r-s, s} \\
--- & --- \\
Z_{s, r-s} & A_{s}
\end{array}\right]: K_{r-s} \in \mathrm{GL}(r-s, 2), A_{s} \in \operatorname{Aut}\left(H_{s}\right)\right\} .
$$

Proposition 4. It holds that

$$
\left|\Gamma_{r}\right|=\left(2^{r-s}-1\right)\left(2^{r-s}-2\right) \ldots\left(2^{r-s}-2^{r-s-1}\right)\left|\operatorname{Aut}\left(H_{s}\right)\right| 2^{(r-s) s} .
$$

Proof: Note that $|\mathrm{GL}(n, 2)|=\left(2^{n}-1\right)\left(2^{n}-2\right) \ldots\left(2^{n}-2^{n-1}\right)$. Also, there are $2^{\ell m}$ distinct matrices $T_{\ell, m}$. 
Theorem 5. The matrix set $\Gamma_{r}$ is a subgroup of $\operatorname{Aut}\left(H_{r}\right)$.

Proof: Let $\left[\begin{array}{c}b_{r-s}^{(u)} \\ --- \\ h_{j}\end{array}\right], u \in\left\{0, \ldots, 2^{r-\mathrm{s}}-1\right\}, j \in\left\{1, \ldots, n_{s}\right\}$, be a column of $H_{r}$ of

(12). Let $M_{r}=\left[\begin{array}{c|c}K_{r-s} & T_{r-s, s} \\ ---- & --- \\ Z_{s, r-s} & A_{s}\end{array}\right] \in \Gamma_{r}$. Then

$$
\left[\begin{array}{c|c}
K_{r-s} & T_{r-s, s} \\
---- & --- \\
Z_{s, r-s} & A_{s}
\end{array}\right]\left[\begin{array}{c}
b_{r-s}^{(u)} \\
-- \\
h_{j}
\end{array}\right]=\left[\begin{array}{c}
K_{r-s} b_{r-s}^{(u)}+T_{r-s, s} h_{j} \\
------ \\
A_{s} h_{j}
\end{array}\right] \in H_{r} .
$$

Moreover, $\operatorname{Det}\left(M_{r}\right)=\operatorname{Det}\left(K_{r-s}\right) \cdot \operatorname{Det}\left(A_{s}\right) \neq 0$, so $\Gamma_{r} \subset \operatorname{Aut}\left(H_{r}\right)$. Finally,

$$
\left[\begin{array}{c|c|c}
K_{r-s}^{\prime} & T_{r-s, s}^{\prime} \\
---- & --- \\
Z_{s, r-s} & A_{s}^{\prime}
\end{array}\right]\left[\begin{array}{cc|c}
K_{r-s}^{\prime \prime} & T_{r-s, s}^{\prime \prime} \\
---- & --- \\
Z_{s, r-s} & \mid & A_{s}^{\prime \prime}
\end{array}\right]=\left[\begin{array}{c|c}
K_{r-s}^{\prime} K_{r-s}^{\prime \prime} & K_{r-s}^{\prime} T_{r-s, s}^{\prime \prime}+K_{r-s}^{\prime \prime} T_{r-s, s}^{\prime} \\
---- & ---------- \\
Z_{s, r-s} & \mid
\end{array}\right] \in \Gamma_{r} .
$$

In general, $\Gamma_{r} \neq \operatorname{Aut}\left(H_{r}\right)$. For example, if we apply repeatedly doubling construction starting from matrix M (so, $s=2$ ), the columns of $H_{r}$ form a $2^{r-1}$-cap of $\mathrm{PG}(r-1,2)$ that is the complement of a hyperplane; its stabilizer group is $\operatorname{AGL}(r-1,2)$ and $|\operatorname{AGL}(r-1,2)|=\left(2^{r}-2\right) \ldots\left(2^{r}-2^{r-1}\right)$. Note that the mentioned cap corresponds to the $\left[2^{r-1}, 2^{r-1}-r, 4\right]$ extended Hamming code.

On the other hand, there exist codes of redundancy $\mathrm{r}$ obtained by doubling construction whose automorphism group is $\Gamma_{r}$.

Lemma 2. Let $X=\left\{x_{1}, \ldots, x_{n}\right\}$ be a set of $n$ boolean values. Let $\Sigma_{n}$ be the multiset of all possible $2^{n}$ sums of elements of $X$ (counting also the sum without addends and attributing the value 0 to it). If at least one of the elements of $X$ is equal to 1 then $\Sigma_{n}$ contains $2^{n-1}$ zeros and $2^{n-1}$ ones.

Proof: By induction on $n$. The case $n=1$ is trivial. In the general case consider the $2^{n-1}$ sums that do not contain $x_{n}$. If an index $i, 1 \leq i \leq n-1$, exists such that $x_{i}=1$, then by the inductive hypothesis $2^{n-2}$ sums are equal to 0 and $2^{n-2}$ sums are equal to 1 . Adding $x_{n}$ we obtain other $2^{n-2}$ sums equal to 0 and $2^{n-2}$ sums equal to 1 whether $x_{n}=0$ or $x_{n}=1$. If $x_{i}=0, i=1, \ldots, n-1$, then the $2^{n-1}$ sums not containing $x_{n}$ are equal to $0, x_{n}=1$ and the $2^{n-1}$ sums containing $x_{n}$ are equal to 1 .

Theorem 6. Let $C_{s}$ be an $\left[n_{s}, n_{s}-s\right]$ code having a parity check matrix $H_{s}$ without zero columns and without rows of weight $n_{s} / 2$. Then for the code $C_{r}$ obtained applying doubling construction $r-s$ times starting from $H_{s}$, it holds that $\operatorname{Aut}\left(C_{r}\right)=\Gamma_{r}$.

Proof: Let $\ell=r-s$. Let $H_{s}=\left[h_{1} \ldots h_{n_{s}}\right]$ where $h_{i}$ is an $s$-positional column. By Lemma $1, H_{r}$ of the form (12) is a parity check matrix of the code $C_{r}$. Let 


$$
M_{r}=\left[\begin{array}{ccc} 
& & t_{1} \\
K_{\ell} & \mid & \vdots \\
& & t_{\ell} \\
------ & \mid & --- \\
x_{\ell+1,1} \ldots x_{\ell+1, \ell} & & a_{1} \\
\vdots & \mid & \vdots \\
x_{r, 1} \ldots x_{r, \ell} & & a_{s}
\end{array}\right] \in \operatorname{Aut}\left(C_{r}\right),
$$

where $K_{\ell}$ is an $\ell \times \ell$ matrix, $t_{i}$ and $a_{j}$ are s-positional rows, and $x_{i, j} \in\{0,1\}$. Let $r_{j}$ be the $j$-th row of $M_{r} H_{r}, j=\ell+1, \ldots, r$. Then

$$
\begin{gathered}
r_{j}=\left[a_{j-\ell} h_{1}^{\mathrm{T}} \ldots a_{j-\ell} h_{n_{s}}^{\mathrm{T}}\left|x_{j, 1}+a_{j-\ell} h_{1}^{\mathrm{T}} \ldots x_{j, 1}+a_{j-\ell} h_{n_{s}}^{\mathrm{T}}\right| x_{j, 2}+a_{j-\ell} h_{1}^{\mathrm{T}} \ldots x_{j, 2}+a_{j-\ell} h_{n_{s}}^{\mathrm{T}} \mid \ldots\right. \\
\left.\ldots \mid x_{j, 1}+\ldots+x_{j, \ell}+a_{j-1} h_{1}^{\mathrm{T}} \ldots x_{j, 1}+\ldots+x_{j, \ell}+a_{j-\ell} h_{n_{s}}^{\mathrm{T}}\right] .
\end{gathered}
$$

As $M_{r} \in \operatorname{Aut}\left(C_{r}\right)$, it induces a permutation on the coordinates of the codewords, so

$$
\text { weight }\left(r_{j}\right)=\operatorname{weight}\left(q_{j}\right)=2^{\ell} \cdot \operatorname{weight}\left(p_{j-\ell}\right),
$$

where $q_{j}$ is the $j$-th row of $H_{r}$ and $p_{i}$ is the $i$-th row of $H_{s}$. On the other hand, fix a value $i, 1 \leq i \leq n_{s}$, and consider the elements of $r_{j}$ in positions $i+(k-1) n_{s}, k=1, \ldots$, $2^{\ell}$, they are: $a_{j-\ell} h_{i}^{\mathrm{T}}, x_{j, 1}+a_{j-\ell} h_{i}^{\mathrm{T}}, x_{j, 2}+a_{j-\ell} h_{i}^{\mathrm{T}}, \ldots, x_{j, 1}+x_{j, 2}+a_{j-\ell} h_{i}^{\mathrm{T}}, \ldots, x_{j, 1}+\ldots+$ $+x_{j, \ell}+a_{j-\ell} h_{i}^{\mathrm{T}}$. All possible sums of elements of the set $\left\{x_{j, 1}, \ldots, x_{j, \ell}\right\}$ appear as addends of $a_{j-\ell} h_{i}^{\mathrm{T}}$. If at least one of the $x_{j, t}$, is equal to 1 , then, by Lemma 2, exactly $2^{\ell-1}$ of these sums are equal to 1 , and therefore exactly $2^{\ell-1}$ of these elements of $r_{j}$ are equal to 1 . It implies weight $\left(r_{j}\right)=n_{s} 2^{\ell-1}$ and weight $\left(p_{j-\ell}\right)=\operatorname{weight}\left(r_{j}\right) / 2^{\ell}=n_{s} / 2$. This is not possible by hypothesis. Moreover, $x_{\ell+1,1}=\ldots=x_{\ell+1, \ell}=\ldots=x_{r, 1}=\ldots=x_{r, \ell}=0$ implies $\operatorname{Det}\left(K_{\ell}\right) \neq 0$, otherwise $\operatorname{Det}\left(M_{r}\right)=0$.

Finally, we show that the $s^{\times} s$ submatrix

$$
A_{s}=\left[\begin{array}{c}
a_{1} \\
\vdots \\
a_{s}
\end{array}\right]
$$

permutes the columns of $H_{s}$, i.e., it belongs to $\operatorname{Aut}\left(C_{s}\right)$. In fact, let $\left[\begin{array}{c}b_{r-s}^{(u)} \\ --- \\ h_{j}\end{array}\right]$, $u \in\left\{0, \ldots, 2^{r-\mathrm{s}}-1\right\}, j \in\left\{1, \ldots, n_{s}\right\}$, be a column of $H_{r}$ of (12). Then, taking into account that $x_{i, j}=0$ in $M_{r}$ of (14), we have

$$
M_{r}\left[\begin{array}{c}
b_{r-s}^{(u)} \\
--- \\
h_{j}
\end{array}\right]=\left[\begin{array}{c}
y \\
- \\
A_{s} h_{j}
\end{array}\right],
$$


where $y$ is an $(r-s)$-positional column. The column $\left[\begin{array}{c}y \\ - \\ A_{s} h_{j}\end{array}\right]$ is a column of $H_{r}$ if and only if $A_{s} h_{j}$ is a column of $H_{s}$. Moreover, if $A_{s} h_{i}=A_{s} h_{j}, i \neq j$, then the $2^{\ell+1}$ columns $\left[\begin{array}{c}b_{r-s}^{(u)} \\ --- \\ h_{i}\end{array}\right],\left[\begin{array}{c}b_{r-s}^{(u)} \\ - \\ h_{j}\end{array}\right], u=0, \ldots, 2^{\ell}-1$, can have only $2^{\ell}$ different images under $M_{r}$.

Corollary 2. Let $C_{s}$ be an $[n, n-s]$ code having a parity check matrix $H_{s}$ without zero columns. If $n$ is odd then for the code $C_{r}$ obtained applying doubling construction $r-s$ times starting from $H_{s}$, it holds that $\operatorname{Aut}\left(C_{r}\right)=\Gamma_{r}$.

By computer search, we obtained the following proposition.

Proposition 5. For the matrices of (2), (8)-(10), it holds that $|\operatorname{Aut}(S)|=120,|\operatorname{Aut}(\Omega)|=336,\left|\operatorname{Aut}\left(\Phi_{1}\right)\right|=40320,\left|\operatorname{Aut}\left(\Phi_{2}\right)\right|=576,\left|\operatorname{Aut}\left(\Phi_{3}\right)\right|=384$, $\left|\operatorname{Aut}\left(\Phi_{4}\right)\right|=720,\left|\operatorname{Aut}\left(\Phi_{5}\right)\right|=11520$.

Corollary 3. Let the value of $\left|\operatorname{Aut}\left(\Phi_{j}\right)\right|$ be as in Proposition 5. It holds that

$$
\begin{gathered}
\left|\operatorname{Aut}\left(\Pi_{r}\right)\right|=120 \cdot 2^{4(r-4)} \prod_{i=0}^{r-3}\left(2^{r-4}-2^{i}\right), \\
\left|\operatorname{Aut}\left(W_{r}\right)\right|=336 \cdot 2^{5(r-5)} \prod_{i=0}^{r-4}\left(2^{r-5}-2^{i}\right), \\
\left|\operatorname{Aut}\left(V_{r, \mathrm{j}}\right)\right|=\left|\operatorname{Aut}\left(\Phi_{j}\right)\right| 2^{6(r-6)} \prod_{i=0}^{r-5}\left(2^{r-6}-2^{i}\right), j=1, \ldots, 5 .
\end{gathered}
$$

Proof: Taking into account that all matrices of (2), (8)-(10), have an odd number of columns, the assertion follows from Proposition 4, Corollary 2, and Proposition 5.

\section{Properness and $t$-properness for error detection of codes obtained by doubling construction}

Problems connected with error detection are considered, e.g., in [3, 8-11, 13], see also the references therein. Here we consider a binary symmetric channel.

Let $p$ be the symbol error probability of the channel.

For the code $C$, let $P_{u e}(C, p)$ be the probability of undetected error under the condition that the code is used only for error detection.

For the code $C$, let $P_{u e}^{(t)}(C, p)$ be the probability of undetected error under the conditions that $d \geq 2 t+1$ and the code is used for correction of $\leq t$ errors.

Definition 3 [8-11]. (i) A binary code $C$ is proper (respectively $t$-proper) if $P_{u e}(C, p)$ (respectively $\left.P_{u e}^{(t)}(C, p)\right)$ is an increasing function of $p$ in the interval $[0,1 / 2]$. 
(ii) Let $a \geq 0$ and $b \leq 1 / 2$ be real values. A binary code $C$ is proper (respectively $t$-proper) in the interval $[a, b]$ if $P_{u e}(C, p)$ (respectively $\left.P_{u e}^{(t)}(C, p)\right)$ is an increasing function of $p$ in $[a, b]$.

Using the results of this work, in particular Theorem 3 and Proposition 3, and papers [2, 3, 8-11], we proved a number of results on the properness and $t$-properness of codes obtained by doubling construction.

Theorem 7 [11, Theorem 2]. Let a binary code of length $n$ have dual distance $d^{\perp}$. If

then the code is proper in the interval

$$
\left\lceil\frac{n}{3}\right\rceil+1 \leq d^{\perp} \leq\left\lfloor\frac{n}{2}\right\rfloor,
$$

$$
\left[\frac{n+1-2 d^{\perp}}{n-d^{\perp}}, \frac{1}{2}\right]
$$

Lemma 3. In doubling construction (1), let the starting $\left[n_{r-1}, n_{r-1}-(r-1), d_{r-1}\right]$ code, given by the parity check matrix $H_{r-1}$, have dual distance $d_{r-1}^{\perp}$ in the region

$$
\left\lceil\frac{n_{r-1}}{3}\right\rceil+1 \leq d_{r-1}^{\perp} \leq\left\lfloor\frac{n_{r-1}}{2}\right\rfloor \text {. }
$$

Then the resultant $\left[n_{r}, n_{r}-r, d_{r}\right]$ code, given by the parity check matrix $H_{r}$, has dual distance $d_{r}^{\perp}$ in the region

$$
\left\lceil\frac{n_{r}}{3}\right\rceil+1 \leq d_{r}^{\perp} \leq\left\lfloor\frac{n_{r}}{2}\right\rfloor
$$

Proof: By (1) and (11), we has $n_{r}=2 n_{r-1}$ and $d_{r}^{\perp}=2 d_{r-1}^{\perp}$.

The right inequality of (15) corresponds to either $2 d_{r-1}^{\perp} \leq n_{r-1}$ (if $n_{r-1}$ is even), or $2 d_{r-1}^{\perp} \leq n_{r-1}-1$ (if $n_{r-1}$ is odd). The right inequality of (16) always corresponds to $2 d_{r-1}^{\perp} \leq n_{r-1}$. So, for all values of $n_{r-1}$, the right part of (16) follows from the right part of (15).

The left inequality of (15) (respectively of (16)) corresponds to one of three cases:

- $n_{r-1}+3 \leq 3 d_{r-1}^{\perp}\left(\right.$ respectively $\left.n_{r-1}+1.5 \leq 3 d_{r-1}^{\perp}\right)$ if $n_{r-1} \equiv 0(\bmod 3)$;

- $n_{r-1}+5 \leq 3 d_{r-1}^{\perp}\left(\right.$ respectively $\left.n_{r-1}+2 \leq 3 d_{r-1}^{\perp}\right)$ if $n_{r-1} \equiv 1(\bmod 3)$;

- $n_{r-1}+4 \leq 3 d_{r-1}^{\perp}\left(\right.$ respectively $\left.n_{r-1}+2.5 \leq 3 d^{{ }^{\perp}}{ }_{r-1}\right)$ if $n_{r-1} \equiv 2(\bmod 3)$.

So, for all values of $n_{r-1}$, the left part of (16) follows from the left part of (15).

Theorem 8. The codes $\Pi_{r}, V_{r, 4}$, and $V_{r, 5}$, are proper in intervals $[a, 1 / 2]$, where

$$
\Pi_{r}^{\perp}: a=\frac{1}{3}+\frac{1}{3 \cdot 2^{r-4}}, r \geq 6 ; \quad V_{r, 4}: a=\frac{5}{11}+\frac{1}{11 \cdot 2^{r-6}}, r \geq 8 ;
$$




$$
V_{r, 5}: a=\frac{3}{10}+\frac{1}{10 \cdot 2^{r-6}}, r \geq 6 .
$$

Proof: We use Proposition 3, Theorem 7, and Lemma 3.

Proposition 6 [11, Remark 1]. An $[n, n-r, d]$ code is proper in the interval $\left[0, \frac{d}{n}\right]$.

Proposition 7. The codes $\Pi_{r}^{\perp}, W_{r}^{\perp}, V_{r, j}^{\perp}$ dual to the codes $\Pi_{r}, W_{r}, V_{r, j}$, are proper in intervals $[0, b]$, where

$$
\begin{gathered}
b=\frac{2}{5} \text { for } \Pi_{r}^{\perp}, b=\frac{2}{9} \text { for } W_{r}^{\perp}, b=\frac{2}{17} \text { for } V_{r, 1}^{\perp}, b=\frac{4}{17} \text { for } V_{r, 2}^{\perp}, \\
b=\frac{5}{17} \text { for } V_{r, 3}^{\perp}, b=\frac{6}{17} \text { for } V_{r, 4}^{\perp}, b=\frac{7}{17} \text { for } V_{r, 5 .}^{\perp} .
\end{gathered}
$$

Proof: We use Propositions 3 and 6.

\section{Definition 4 [8-10].}

- Let $C$ be an $[n, n-r, d]$ code with dual weight spectrum $\left\{A_{0}^{\perp}, \ldots, A_{n}^{\perp}\right\}$. Dual extended binomial moment $B_{\ell}^{*}$ is defined as follows:

$$
B_{\ell}^{*}=\frac{1}{\left(\begin{array}{l}
n \\
\ell
\end{array}\right)} \sum_{i=1}^{\ell}\left(\begin{array}{c}
n-i \\
n-\ell
\end{array}\right) A_{i}^{\perp}, \ell=1, \ldots, n .
$$

- Let $C$ be an $[n, n-r, d]$ code. Let $Q_{h, i}$ be the number of vectors of weight $i$ in the cosets of weight $h$, excluding the coset leaders. We define the following values:

$$
A_{\ell, t}^{*}=\frac{1}{\left(\begin{array}{l}
n \\
\ell
\end{array}\right)} \sum_{i=t+1}^{\ell}\left(\begin{array}{c}
n-i \\
n-\ell
\end{array}\right) \sum_{h=0}^{t} Q_{h, i}, \ell=t+1, \ldots, n .
$$

Theorem 9 [8, Theorem 6]. Let $C$ be an $[n, n-r, d]$ binary code with dual distance $d^{\perp}$ and dual extended binomial moments $\left\{B_{1}^{*}, \ldots, B_{n}^{*}\right\}$. Let $d+d^{\perp} \leq n$. If

then $C$ is proper.

$$
B_{n-\ell}^{*} \leq B_{n-\ell+1}^{*}-2^{r-\ell}, \ell=d+1, \ldots, n-d^{\perp}+1,
$$

Proposition 8. The codes with the parity check matrices $S$ and $\Omega$ are proper.

The codes $\Pi_{r}$ with $r=5,6,7,8,9$ are proper. The code $W_{6}$ is proper.

Proof: We use Proposition 3 and Theorem 9.

Proposition 9. The codes $\Pi_{r}$ with $10 \leq r \leq 20$ are not proper.

Proof: Using [9, Equation (2.2)] and Proposition 3, we obtain

$$
\begin{gathered}
P_{u e}\left(\Pi_{r}, p\right)=\frac{1}{2^{r}}\left(1+10(1-2 p)^{2^{r-3}}+\left(2^{r}-16\right)(1-2 p)^{5 \cdot 2^{r-5}}+\right. \\
\left.+5(1-2 p)^{2^{r-2}}\right)-(1-p)^{5 \cdot 2^{r-4}} .
\end{gathered}
$$

The corresponding derivative by $p$ is 


$$
\begin{gathered}
P^{\prime}{ }_{u e}\left(\Pi_{r}, p\right)=5\left(-\frac{1}{2}(1-2 p)^{2^{r-3}-1}-\left(2^{r-4}-1\right)(1-2 p)^{5 \cdot 2^{r-5}-1}-\frac{1}{2}(1-2 p)^{2^{r-2-1}}+\right. \\
\left.+2^{r-4}(1-p)^{5 \cdot 2^{r-4}-1}\right) .
\end{gathered}
$$

Taking into account Theorem 8 , we checked by computer that, for $10 \leq r \leq 20$, in the region $\left(0, \frac{1}{3}+\frac{1}{3 \cdot 2^{r-4}}\right)$ there exist values of $p$ such that the derivative $P^{\prime}{ }_{u e}\left(\Pi_{r}, p\right)$ is negative.

Theorem 10 [10, Theorem 2]. Let $C$ be an $[n, n-r, d]$ binary code with $A_{\ell, t}^{*}$ as in (17). If

\section{then $C$ is $t$-proper.}

$$
A_{\ell, t}^{*}-2 A_{\ell-1, t}^{\star} \geq 0, \ell=t+2, \ldots, n,
$$

Proposition 10. The codes with the parity check matrices $S$ and $\Omega$ are 1-proper. The codes $\Pi_{r}$ with $r=5,6,7$ are 1-proper. The code $W_{6}$ is 1-proper.

Proof: We use Theorem 10. In order to calculate the values of $A_{\ell, t}^{*}$, we take the parity check matrices of the corresponding codes.

Acknowledgements: The research of A. A. Davydov was carried out at the IITP RAS at the expense of the Russian Foundation for Sciences (project 14-50-00150). The research of S. Marcugini and F. Pambianco was supported in part by Ministry for Education, University and Research of Italy (MIUR) (Project "Geometrie di Ga-lois e strutture di incidenza") and by the Italian National Group for Algebraic and Geometric Structures and their Applications (GNSAGA-INDAM).

\section{References}

1. A f a n a s s i e v, V. B., A. A. D a v y d o v. Weight Spectrum of Quasi-Perfect Binary Codes with Distance 4. - In: Proc. of IEEE Int. Symp. Inform. Theory (ISIT’17), Aachen, Germany, 2017, pp. 2193-2197.

\section{http://ieeexplore.ieee.org/document/8006918/}

2. A f a n a s s i e v, V. B., A. A. D a v y d o v, D. K. Z i g a n g i r o v. Design and Analysis of Codes with Distance 4 and 6 Minimizing the Probability of Decoder Error. - J. Communic. Technology Electronics, Vol. 61, 2016, No 12, pp. 1440-1455.

3. B a i c h e va, T., S. D o d u n e k o v, P. K a z a k o v. On the Undetected Error Probability Performance of Cyclic Redundancy-Check Codes of 16-bit Redundancy. - IEEE Trans. Comm., Vol. 147, 2000, No 5, pp. 253-256.

4. B a r t o l i, D., S. M a r c u g i n i, F. P a m b i a n c o. A Computer Based Classification of Caps in PG(5, 2). arXiv:1203.0994 [math.CO], 2012.

5. D a v y d o v, A. A., S. M a r c u g i n i, F. P a m b i a n c o. Further Results on Binary Codes Obtained by Doubling Construction. - In: Proc. Eighth International Workshop on Optimal Codes and Related Topics, OC'17 (in Second International Conference "Mathematics Days in Sofia"), Sofia, Bulgaria, 2017, pp. 73-80.

6. D a v y d o v, A. A., L. M. T o m b a k. Quasiperfect Linear Binary Codes with Minimal Distance 4 and Complete Caps in Projective Geometry. - Problems Inform. Transm., Vol. 25, 1989, No 4, pp. 265-275.

7. D a v y d o v, A. A., L. M. T o m b a k. An Alternative to the Hamming Code in the Class of SECDED Codes in Semiconductor Memory. - IEEE Trans. Inform. Theory, Vol. IT-37, 1991, No 3, pp. 897-902. 
8. D o d u n e k o v a, R. Extended Binomial Moments of a Linear Code and the Undetected Error Probability. - Problems Inform. Transm., Vol. 39, 2003, No 3, pp. 255-265.

9. D o d u n e k o v a, R., S. M. D o d u n e k o v, E. N i k o lov a. A Survey on Proper Codes. Discrete Appl. Math., Vol. 156, 2008, No 9, pp. 1499-1509.

10. D o d u n e k o v a, R., S. M. D o d u n e k o v. $t$-Good and $t$-Proper Linear Error Correcting Codes. - Mathematica Balkanica. New Series, Vol. 17, 2003, No 1-2, pp.147-154.

11. D o d u n e k o v a, R., E. N i k o 1 o v a. Sufficient Conditions for Monotonicity of the Undetected Error Probability for Large Channel Error Probabilities. - Probl. Inform. Transm., Vol. 41, 2005, No 3, pp. 187-198.

12. K h a t i r i n e j a d, M., P. Li s o n e k. Classification and Constructions of Complete Caps in Binary Spaces. - Des. Codes Cryptogr., Vol. 39, 2006, No 1, pp. 17-31.

13. K $1 \varnothing \mathrm{v}$ e, T. Codes for Error Detection. Singapore, World Scientific, 2007.

14. M a c W illi a m s, F. J., N. J. A. S 1 o a n e. The Theory of Error-Correcting Codes. North-Holland, Amsterdam, 1977.

15. P a n c h e n k o, V. I. On Optimization of Linear Code with Distance 4. - In: Proc. of 8th All-Union Conf. on Coding Theory and Communications, Kuibyshev, 1981, Part 2: Coding Theory, Moscow, 1981, pp. 132-134 (in Russian). 